\title{
Issues with the Construct of Quality
}

\author{
Nele Van den Ende ${ }^{1}$, Jettie \\ Hoonhout $^{1}$ \& Lydia Meesters ${ }^{2}$ \\ ${ }^{1}$ Philips Research \\ HTC 34, NL - 5656AE Eindhoven \\ ${ }^{2}$ Eindhoven University of \\ Technology \\ Den Dolech 2 \\ $\mathrm{NL}$ - 5612AZ Eindhoven \\ \{nele.van.den.ende, \\ jettie.hoonhout\}@philips.com \\ I.m.j.meesters@tue.nl
}

\begin{abstract}
This paper proposes an outline for a framework that aims to give a comprehensive view of perceived video quality, including physical characteristics, perceptual attributes and cognitive factors.
\end{abstract}

\section{Keywords}

theoretical framework, quality research, perceived video quality

\section{INTRODUCTION}

When testing subjective video quality, it is relatively easy to manipulate or vary physical characteristics and ask people for a response. This response should then be straightforward and relatively easy to interpret. Unfortunately, things are not as easy and straightforward as that. For example, perceptual attributes of video such as blockiness and jerkiness can influence each other, but the influence of attribute A on B is not necessarily the same as the influence of attribute B on A.

An example might help to clarify why perceived quality is not as straightforward as it may seem: think of the quality of food, say tomatoes. The way tomatoes are displayed in a groceryshop influences your memories and associations of their taste: all jumbled together, they might have blemishes, but lined up like soldiers they could be prime quality. Then there are people who do not like tomatoes: no matter the way tomatoes are displayed, they will still not like them and will probably not be inclined to sample them. Another part is that a nice big red tomato can make you think that it is juicier and tastier than its neighbor, which is not as big and not a nice equal red. Interaction can be found as well, because tomatoes go very well together but basil, but most people would probably think twice before eating peanut-butter and tomatoes.

For video quality, there are similar problems. When watching multimedia, people might not like the shown video sequences and therefore judge the video quality as lower than someone

(C) N. Van den Ende et al, 2007

Published by the British Computer Society

Volume 2 Proceedings of the 21st BCS HCI Group Conference HCI

2007, 3-7 September 2007, Lancaster University, UK

Devina Ramduny-Ellis \& Dorothy Rachovides

(Editors) who does like them. A good question that has been used for

quite some time is to ask "What is video quality?". What do experts who ask about video quality mean, and what do they expect from it? What do they intend video quality to mean to laypeople? We postulate that "video quality" is often used to indicate an internally represented construct. This internal representation is, among other things, influenced by experiences and can fluctuate over time.

\section{FRAMEWORK OUTLINE}

Janssen [4] has defined image quality as a useful attribute of an image, which expresses how well the observer is able to employ the image as a source of information about the outside world. So the quality of an image is determined by the adequacy with which the image can serves as input to visual perception. Specifically, the adequacy of said image as input to visual perception is determined through discriminability and identifiability of items depicted in said image. There are other image quality models that it would be possible to continue with, but video is another context, which does not always have the same applications and uses.

Gulliver \& Ghinea [3] devised a framework where Quality is divided between Quality of service, which focuses only on technical aspects, and Quality of Perception (QoP), which focuses on the subjective level of quality. QoP uses the level of information transfer and user satisfaction to find out subjective opinions. User satisfaction especially asks about two different things: presentation quality independent of content and enjoyment of multimedia content. Here, quality is already determined on several levels, but there are only 3 cognitive constructs that are allowed to explicitly contribute to user judgment.

Visual perception, from the human visual system to cognitive process, is not entirely understood yet. A lot is known about the early stages of human visual perception, from the light going in to the retina, from the cells discriminating between orientation of lines. However, there is no consensus yet about what happens later on in the brain with the interpretation of images [8].

Physical characteristics in the material are not the only influence; there is a top-down interaction to reckon with as well. Top-down interaction is defined here as coming from people their preconceived notions about quality, their expectations, the kind of television they are used to, motivation 
FIGURE 1: outline of the proposed framework.

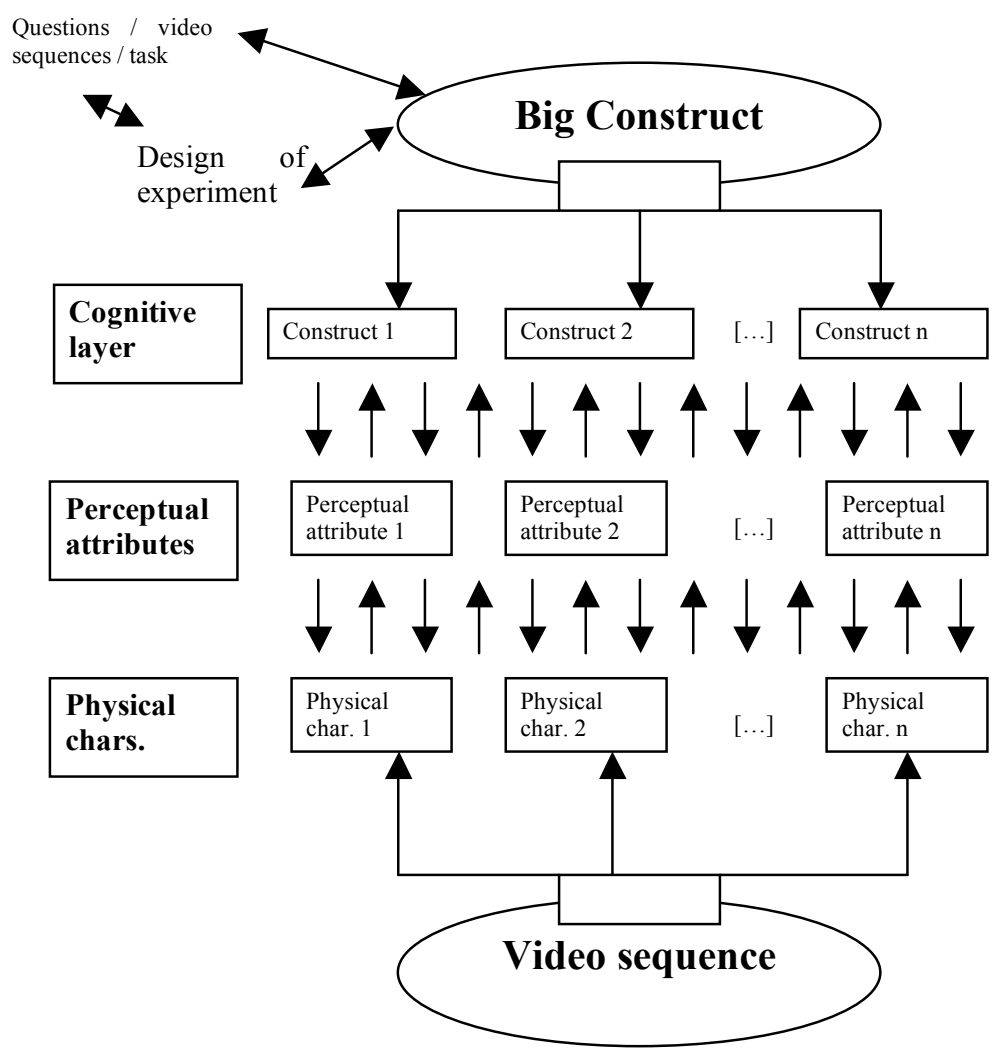

and external information about visual details based on incoming light (in the eye). On top of that humans need focused attention to notice changes in visual scenes.

\section{DISCUSSION AND FUTURE WORK}

Obviously, the framework as outlined in figure 1 is far from finished. A lot of work has been done on the influence of the physical characteristics (such as change in frame rate, quantization levels and chrominance levels,...) and the perceptual attributes (such as jerkiness, blockiness, blurring, ringing, colour bleeding, ...), but the real challenge will be in combining the top-down and bottom-up approach.

Many studies will be needed to provide input to work out this framework in more detail. The first step that we consider next is looking at the influence of involvement on perceived image quality. Involvement of users into the content could be tested by using an experimental and a control group. The control group would see pictures and would have to judge the perceived quality of the pictures. The experimental group would see the same pictures, but in such a way that they form a story and give participants a way to be drawn in the story, to give them an intrinsic motivation for watching and judging perceived image quality. to watch television, attention they are actually giving to the material, .... There seem to be a lot of factors that contribute to subjective video quality and it is currently unclear whether or how these factors influence users' judgment (see figure 1 for a way to look at the interaction between top-down and bottom-up factors).

When testing subjective video quality, there is another "layer" to keep in mind, experimental design. External and internal judgment scales are not comparable, it has been proven that internal judgment scales are influenced by the choice of the rating scale given (do you ask for quality or impairment, and in which way?), in which order the material is offered and video or image content shown [5]. Furthermore, voice quality research has shown that, when given more external benchmarks for the scale (options to listen to what the researchers think is a medium rough voice, for example, and compare it to the given sample), external standards are more constant [2].

So, people device a judgment strategy based on a number of factors, one of which is the experimental procedure itself and another is the video content [1]. In addition to those factors, people tend to watch video and multi-media for a reason: so there is the motivation and attention with which they watch video. Video content also plays a role in deciding whether or not they will continue to watch said video content. Other constructs that could play a role are challenge, engagement, overall enjoyability, future use, situational factors, aesthetics of the CE-application (television, mobile phone, PDA, ...), expectations and presence [7]. These constructs are not easy to test, however. For example, expectations cannot really be assessed after watching video material, because it is likely that they will have changed already. Self-rapportage is not always the best way to research expectations.

Rensink [6] also proposes that visual perception of a scene on a display is related to internal information based on knowledge

\section{ACKNOWLEDGMENTS}

Thanks to Don Bouwhuis, Klaus Kursawe, Matthias Krause and Paul Shrubsole for their support and comments.

\section{REFERENCES}

[1] de Ridder, H.(2001). Cognitive issues in image quality measurements. Journal of Electronic Imaging, vol. 10, no. 1, pp. $47-55$

[2] Gerratt, B. R., Kreiman, J., Antonanzas-Barroso, N., and Berke, G. S.(1993). Comparing Internal and External Standards in Voice Quality Judgments. Journal of Speech and Hearing Research, vol. 36 pp. 14-20, 1993.

[3] Gulliver, S. R. and Ghinea, G. (2006). Defining user perception of distributed multimedia quality. $A C M$ Transactions on Multimedia Computing, Communications, and Applications (TOMCCAP), vol. 2, no. 4, pp. 241-257

[4] Janssen, T. J. W. M. (1999). Computational Image Quality. Eindhoven University of Technology

[5] Meesters, L. M. J. and Martens, J.-B. (2000). Influence of processing method, bit-rate, and scene content on perceived and predicted image quality. 3959, 45-55. SPIE Conference on Human Vision and Electronic Imaging.

[6] Rensink, R. A. (2002) Internal vs. External Information in Visual Perception. 24, 63-70. ACM Press. SMARTGRAPH '02: Proceedings of the 2nd international symposium on Smart graphics.

[7] Schickenberg, B. and Hoonhout, H. C. M.(2003). Development of a rating scale to determine the appeal of enriched content to users. Philips Research

[8] Solso, R. L. (1995) Cognitive Psychology, 3d ed. Boston: Allyn \& Bacon 